\title{
THE FIRST WAKEFIELD TEST ON THE C-BAND CHOKE-MODE ACCELERATING STRUCTURE
}

\author{
T. Shintake, H. Matsumoto, N. Akasaka, M. Yoshida, KEK, Tsukuba, Japan \\ C. Adolphsen, K. Jobe, D. McCormick, M. Ross, T. Slaton, SLAC, Stanford, CA
}

\begin{abstract}
A full-scale prototype C-band structure for the e+e- linear collider has been built in which the dipole powers are damped to suppress the long-range transverse wakefield. To verify that the damping works as expected, the C-band structure was tested in the Accelerator Structure SETup (ASSET) at SLAC. This paper presents results of the wakefield measurement with ASSET.
\end{abstract}

\section{INTRODUCTION}

The C-band scheme being considered at KEK for the 500 $\mathrm{GeV}$ to $1 \mathrm{TeV}$ scale linear collider employs multi-bunch beam operation in a high accelerating gradient C-band linac [1]. If no any cares were taken in the accelerating structure, the high- $Q$ transverse dipole mode, mainly TM110 mode, would produce an enormous blow up the transverse motion of the bunch train, resulting in loosing luminosity. In order to damp all the dipole modes in accelerator cavities, the author proposed the choke-mode cavity in 1992 [2]. In this scheme, the beam excited power is eliminated by a microwave absorbing material in the accelerator, thus the dipole modes are damped in each cell for a wide frequency-range. The choke is used as a notchfilter, which traps the rf power within a cavity at the operating frequency, thus a high accelerating field can be generated.

The basic principle of the choke-mode cavity was proved using an S-band model in 1994, where an electron beam was accelerated at the field-gradient as high as $50 \mathrm{MV} / \mathrm{m}$ driven by S-band power of $100 \mathrm{MW}$ peak.

From 1996, we started design of C-band RF system, and developed a full-scale prototype for the C-band linear collider. The structure was installed in ASSET at SLAC, and its electrical performance was tested with electron/positron bunches in December 1998.

\section{C-BAND STRUCTURE}

Figure 1 shows the developed C-band structure, which is composed of the regular section ( 89 choke-mode cells), input/output couplers attached at each end, and two RFBPMs for beam position alignment at both end. A wakefield monitor is prepared on the center cell in order to observe the beam induced HOM spectrum, and determine the beam position. To avoid unwanted transverse kick due to asymmetric field, the double-feed coupler using $\mathrm{J}$-shaped waveguide is employed in the input/output couplers [3]. The whole structure is enclosed in a cooling-water jacket made by stainless steel.

\subsection{Choke-Mode Cell Design}

Figure 2 shows the cut-view of the C-band structure, including the wakefield monitor at the center cell. The beam-induced wakefield-power is damped in the microwave absorbers made by $\mathrm{SiC}$-ceramic, which is mechanically attached in the copper cell with a metalspring-insert. The design detail is reported in ref. [3].

In order to maximise the damping performance for the lowest dipole mode, the volume of the $\mathrm{SiC}$ ring was optimised using computer simulation [4]. Three different sizes of the $\mathrm{SiC}$ ring were used in the current design. The wakefield of the whole accelerating structure was approximately estimated by averaging the wakefield calculated at equally spaced 12 points. It was expected that the wakefield would be damped below 0.5 $\mathrm{V} / \mathrm{pC} / \mathrm{m} / \mathrm{mm}$ at the second bunch at $2.8 \mathrm{nsec}(0.84 \mathrm{~m})$, which is lower than the limit of $0.7 \mathrm{~V} / \mathrm{pC} / \mathrm{m} / \mathrm{mm}$ defined by the multi-bunch emittance dilution of $25 \%$ in the main linac.

\section{$2.2 R F-B P M$}

In the C-band linear collider, it is necessary to align the accelerating structure with respect to the beam in rather tight tolerances, a high-precision BPM will be attached at the both end of the structure and the transverse positions will be adjusted by fine movers. To limit the emittance dilution below $25 \%$ in the main linac, the structure should be aligned with accuracy better than $30 \mu \mathrm{m}$ and fabricated with straightness within $50 \mu \mathrm{m}$.

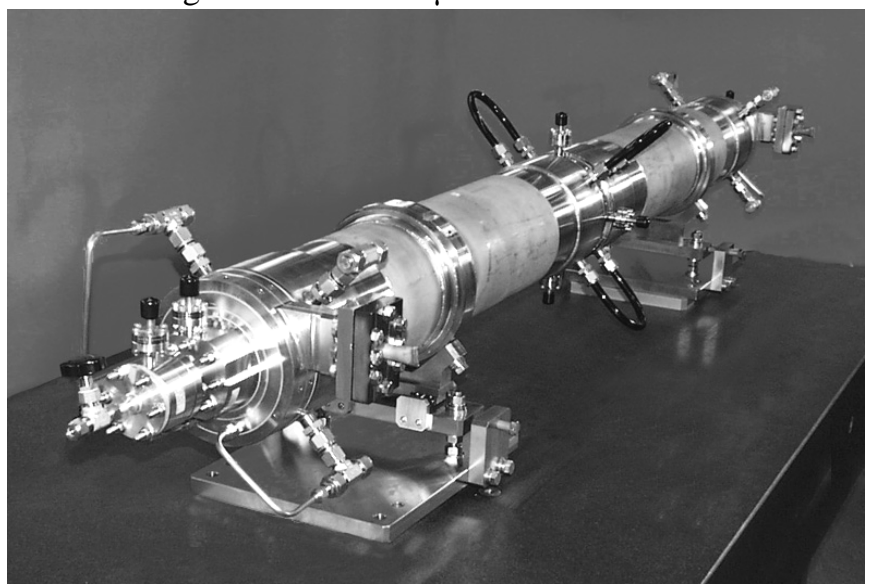

Figure 1: C-band accelerating structure. $2 \mathrm{~m}$ long, 91 cell. 


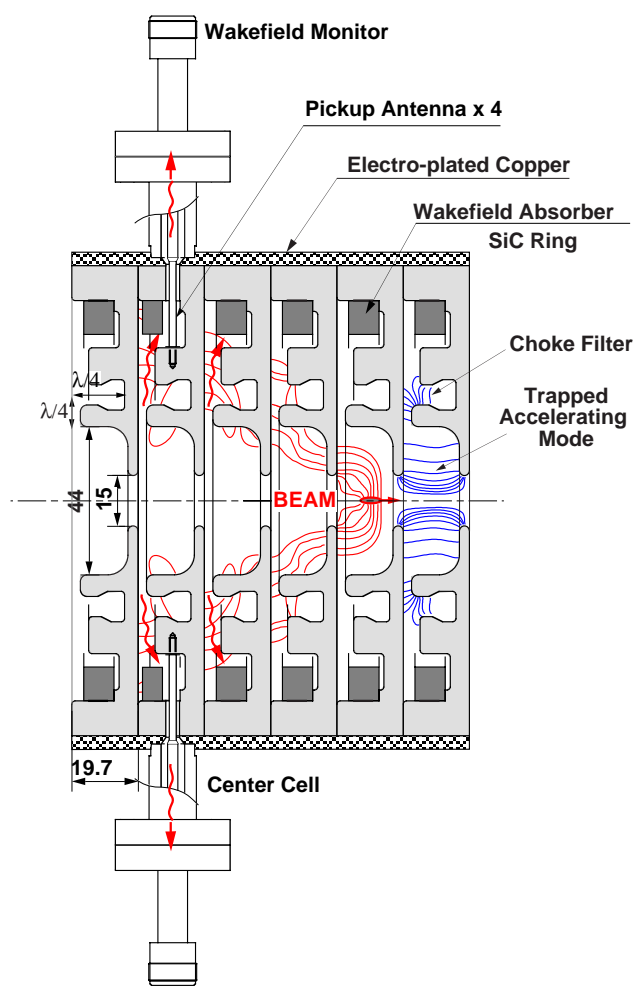

Figure 2: A cut view of the C-band chokemode structure with a wakefield monitor.

For the C-band accelerator alignment, a new type RFBPM was developed as shown in Fig. 3, which uses longitudinal slots to cut the common-mode signal from the beam-position signal. To achieve highly accurate BPM, especially to avoid unwanted shift of the electrical center (zero point), elimination of the common-mode power is the most important key [5]. In this new design, reduction of common mode by $-40 \mathrm{~dB}$ or better can be made.

\section{ASSET BEAM TEST}

\subsection{Wakefield Signal Spectrum}

In order to monitor the microwave signal generated by the beam, four pick-up antennas were prepared on the center cell as shown in Fig. 2. The antenna is the straight metal rod, which forms a closed loop together with the ground plane, and couples with the magnetic field behind the SiC absorber. Figure 4 shows the measured spectrum for the

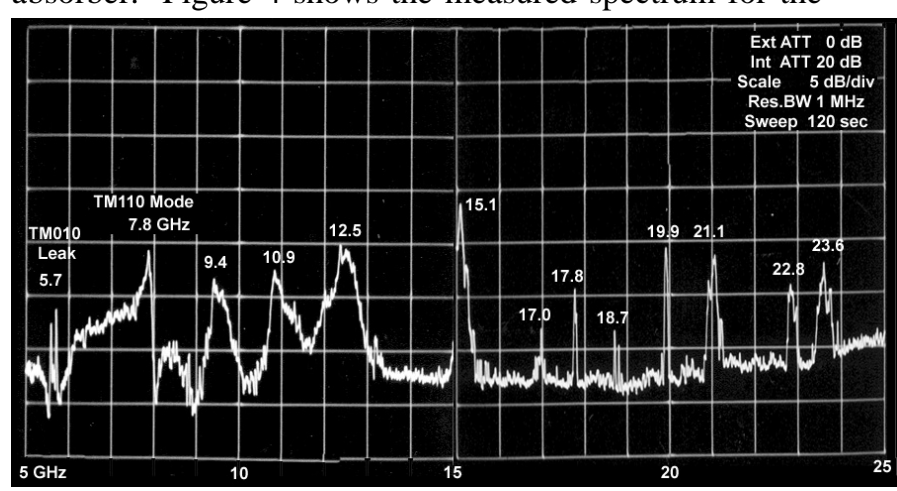

Fig. 4 Measured wakefield spectrum with a horizontal antenna.

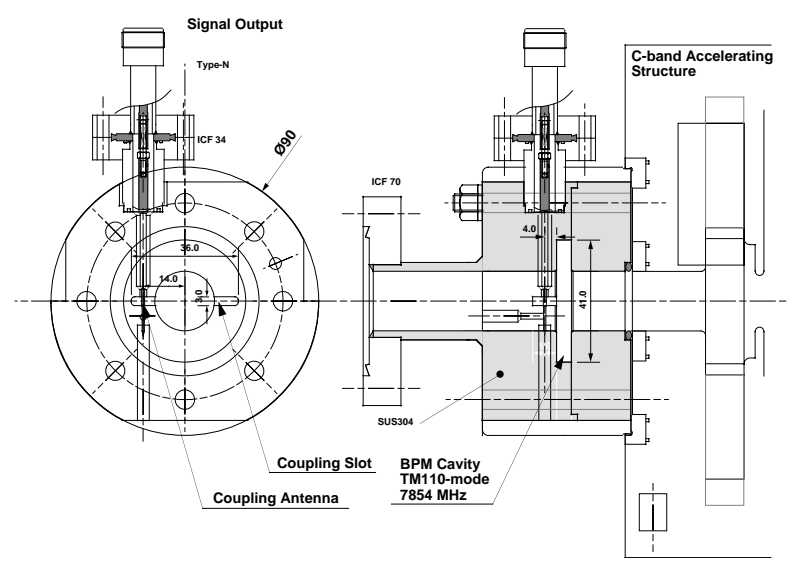

Figure 3: RF-BPM with common-mode less design.

frequency range between 5 to $25 \mathrm{GHz}$. The sharp peaks at $5.7 \mathrm{GHz}$ are the leakage power from the beam excited dominant accelerating mode. The next peak at $7.8 \mathrm{GHz}$ is the lowest dipole mode (TM110 mode). The measured Qfactor was 36 , which is slightly higher than the expected value of 30 estimated with the computer simulation. As shown in Fig. 2, the microwave absorber in the monitor cell has smaller dimension, thus the Q-factor takes higher value than the regular cells. According to the computer simulation, the effective $Q$ for the averaged wakefield is expected as low as 12 .

Most of all peaks at higher frequency bands were identified with the simulated spectrum. Exception was a few sharp peaks around $20-24 \mathrm{GHz}$, which were not predicted at design time. The same peaks appeared as a long tail in the measured wakefield as discussed later.

In Fig. 4, the peak at $15 \mathrm{GHz}$ seems to be the highest one, but its power is much lower than the dominant dipole mode at $7.8 \mathrm{GHz}$. This is due to the characteristic of the pick-up. Since the loop-coupler picks up the timevariation of the magnetic flux:

$$
V \propto d \Phi / d t=\omega B S,
$$

A higher frequency component generates a higher output signal for the same intensity of magnetic field.

\subsection{RF-BPM Test}

Figure 5 shows the circuit diagram of the RF-BPM. The rf signal from two RF-BPMs, the wakefield monitor and the reference cavity are processed with the band-pass filters (7854 MHz, 3\% BW), and down converted to 310 $\mathrm{MHz}$ signal, finally sampled with 4-ch digital scope (Tektronix TDS684B, 5Gs, 1GBW). The reference signal is used to find the beam timing and the beam phase. The wakefield signal from two pick-up antennas at the center BPM are combined in a 180deg. hybrid to eliminate the common mode power.

Figure 6 is the correlation plot for three BPMs. The zero-cross point gives the vertical offset of the center BPM with respect to the mean center of the upstream and downstream BPMs. It was $+63 \mu \mathrm{m}$. By taking account the calibration data, the straightness becomes 


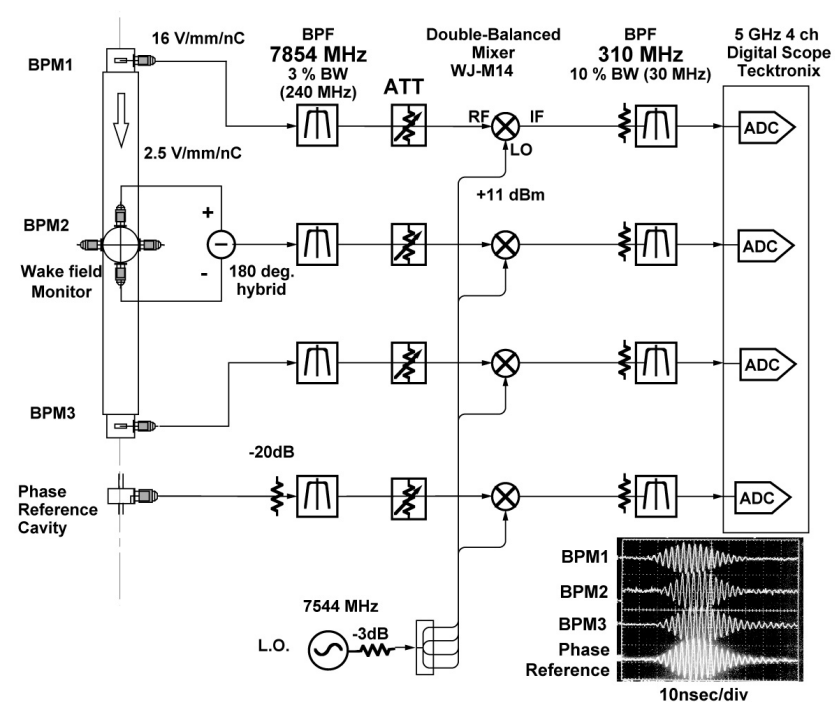

Fig. 5 RF-BPM detector circuit.

$49 \mu \mathrm{m}$. This is just border of design tolerance. On the other hand, the straightness mechanically measured from outside was $90 \mu \mathrm{m}$. We need further study before concluding these test results, such as measurement of the straightness using laser-beam from inside of the structure. The observed spatial resolution was about $8 \mu \mathrm{m}$, which was limited by high digital noise in the sampling-scope.

\subsection{Wakefield Measurement}

We measured the wakefield generated in the C-band structure with the same method as done in the X-band structure R\&D. The layout of the ASSET and detailed procedure are found in elsewhere [6].

We injected the positron beam first as a drive bunch, which extracted from the South Damping Ring. The bunch passed through the C-band structure and was then steered into a dump. Right after the positron, the electron bunch was injected from the North Damping Ring and the deflection angle due to the wakefield was measured with the BPMs in the linac. The measured data is shown in Fig. 7. The measured wakefield damps according to the expected waveform (solid line) up to $1.6 \mathrm{nsec}(0.5 \mathrm{~m})$ from about 15 to $1 \mathrm{~V} / \mathrm{pC} / \mathrm{m} / \mathrm{mm}$. However, after this

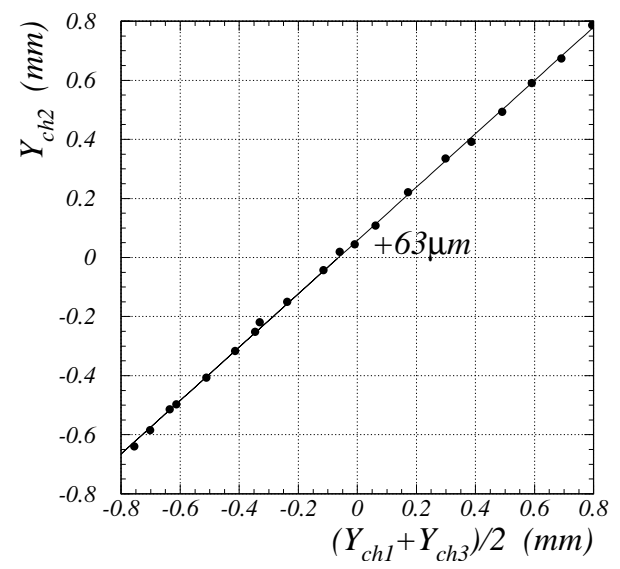

Fig. 6 Correlation plot on three BPM readout.

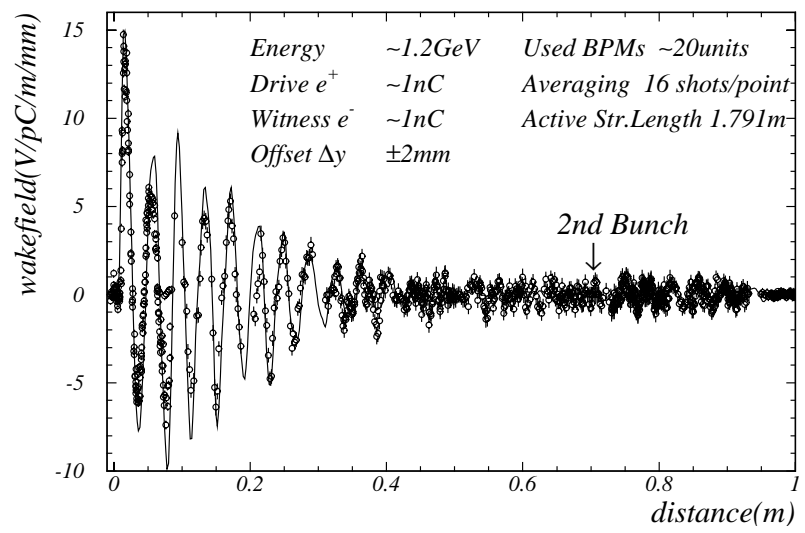

Fig. 7 Measured (circle) and expected (solid line) wakefield.

point, the measured wakefield showed a high frequency oscillation with an amplitude of $0.8 \sim 1.0 \mathrm{~V} / \mathrm{pC} / \mathrm{m} / \mathrm{mm}$. This is just the border-line of the $25 \%$ emittance dilution. Applying FFT, we found that the oscillation corresponds to the spectrum peak at $20,23 \mathrm{GHz}$. With computer simulations, it was found that in some condition the mode can be trapped in the cavity at very high-frequency, whose field pattern has a node-point at the slot location, thus no power can leak out to the damper. This was caused by our design change. To improve the shunt-impedance by about $10 \%$, we changed the disk thickness from 4 to $3 \mathrm{~mm}$. However, the mode pattern in the cavity moved in $\mathrm{z}$ direction, and occasionally created a node at the coupling slot. To solve this, we will shift z-location of the damping slot by $2 \mathrm{~mm}$. With careful simulations, it was shown that the trapped mode could be eliminated. The new model is under design.

\section{ACKNOWLEDGEMENT}

We would like to thank Prof. David Burke for his encouragement, and Charles Yoneda, Leo Giannini, Leif Eriksson, Tony R. King, M. W. Perry, J. R. McDougal for their help in structure installation.

\section{REFERENCES}

[1] T. Shintake et al. ,"C-band RF Main Linac System for e+e- Linear Collider at $500 \mathrm{GeV}$ to $1 \mathrm{TeV}$ C.M. Energy", EPAC96, KEK Preprint 96-68, and K. Yokoya et al., "C-band Linear Collider with C.M. Energy $500 \mathrm{GeV}$ to $1 \mathrm{TeV}$ ",

[2] T. Shintake, "The Choke Mode Cavity", Jpn. J. Appl. Phys. Vol.31, pp. L1567-L1570, Part2, No.11A, 1992

[3] H. Matsumoto, T. Shintake and N. Akasaka, "Fabrication of the Cband $(5712 \mathrm{MHz})$ Choke-Mode Type Damped Accelerator Structure, LINAC98, KEK Pre-print 98-143.

[4] N. Akasaka T. Shintake and H. Matsumoto, "Optimisation on Wakefield Damping in C-band Accelerating Structure", LINAC98, August 23-28, Chicago, IL, KEK Pre-print 98-142

[5] T. Shintake, "Development of Nanometer Resolution RF-BPMs", HEACC98, Sept 1998, Dubna, Russia, KEK Pre-print 98-188.

[6] C. Adolphsen, et al., "Measurement of Wakefield Suppression in a Detuned X-band Accelerator Structure", LINAC94 Proc., p481 